\title{
Liaison Psychiatry in a Tertiary Hospital Centre An Inpatient Based Analysis
}

Gomes-Pereira, E ${ }^{1}$; Machado, AS ${ }^{1}$; Côrte-Real, A ${ }^{1}$; Grangeia, R ${ }^{1}$; Reis, $C^{1}$

${ }^{1}$ Clinic of Psychiatry and Mental Health of São João University Hospital Centre, Porto

\section{Background}

Previous history of psychiatric disorder and de novo psychiatric symptomatology are common among inpatients admitted for non-psychiatric diagnosis. Liaison psychiatry has a fundamental role in the evaluation, management and follow-up of these patients, potentially improving healthcare and reducing hospital length of stay. With that in mind, a more in-depth knowledge of this population may be of great benefit, with a likely positive impact on the quality of care provided, on morbidity reduction and on multidisciplinary work and communication.

\section{Objectives}

We aimed to analyze and describe a cohort of inpatients observed by a Liaison Psychiatry Unit of a tertiary hospital centre.

\section{Methods}

A retrospective and observational study was carried. We revised clinical data from patients observed in psychiatry internal consultation of the Clinic of Psychiatry and Mental Health of São João University Hospital Centre, from August 2018 to January 2019. Statistical analysis with SPSS ${ }^{\circledR}$ v20 was made.

\section{Results}

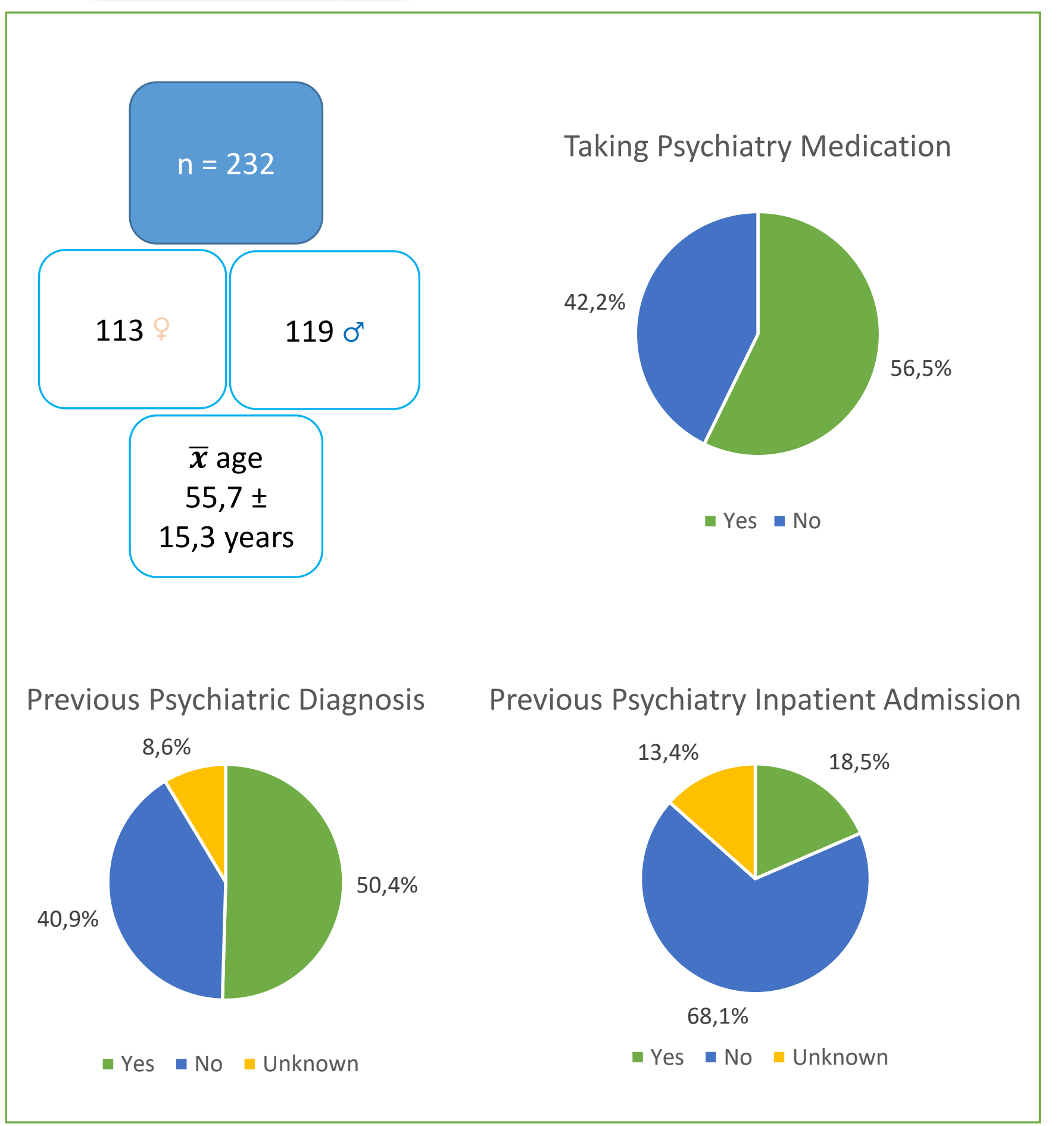

\begin{tabular}{|l|l|}
\hline \multicolumn{1}{|c|}{ Requesting Department } & n (\%) \\
\hline Internal Medicine & $28(12,1 \%)$ \\
\hline Hematology & $24(10,2 \%)$ \\
\hline Infectious Diseases & $23(9,9 \%)$ \\
\hline Pshysical Medicine and Rehabilitation & $17(7,3 \%)$ \\
\hline Intermediate/Intensive Care & $17(7,3 \%)$ \\
\hline Orthopedics & $16(6,9 \%)$ \\
\hline Other & $107(46,3 \%)$ \\
\hline
\end{tabular}

\begin{tabular}{|l|l|}
\hline \multicolumn{1}{|c|}{ Reason for Request } & n (\%) \\
\hline Depressive Symptomatology & $55(23,7 \%)$ \\
\hline Psychomotor Agitation & $35(15,1 \%)$ \\
\hline History of a Previous Psychiatric Diagnosis & $26(11,2 \%)$ \\
\hline Anxious Symptomatology & $14(6,0 \%)$ \\
\hline Treatment Non-adherence & $13(5,6 \%)$ \\
\hline Other & $92(38,4 \%)$ \\
\hline Adjustment Diagnosis & $n(\%)$ \\
\hline Depressive Disorders & $47(20,3 \%)$ \\
\hline Delirium & $44(19 \%)$ \\
\hline No Psychiatric Diagnosis & $30(12,9 \%)$ \\
\hline Substance Use Disorders & $30(12,9 \%)$ \\
\hline Other & $19(8,2 \%)$ \\
\hline
\end{tabular}

\section{Therapeutic Orientation}

Psychiatry medication was:

- Started in $41,8 \%$ of patients ( $n=97)$

- Adjusted in $27,6 \%$ of patients $(n=64)$

- Stopped in $10,8 \%$ of patients $(n=25)$

Psychology support was requested in $\underline{16,8 \%}$ of patients $(n=39)$.

\begin{tabular}{|l|l|}
\hline \multicolumn{1}{|c|}{ Follow-Up After Discharge } & \multicolumn{1}{|c}{$n(\%)$} \\
\hline Primary Care & $93(40,1 \%)$ \\
\hline General Psychiatry Consultation & $69(29,7 \%)$ \\
\hline Liaison Psychiatry Consultation & $31(13,4 \%)$ \\
\hline Psychiatry Inpatient Ward & $3(1,3 \%)$ \\
\hline Psychology Consultation & $3(1,3 \%)$ \\
\hline Other & $24(10,4 \%)$ \\
\hline
\end{tabular}

\section{Other Results}

- Patients with a final diagnosis of Delirium were significantly older than patients with other diagnosis $(64,9 \pm 13,6$ vs. $54,3 \pm 15,0$ years, $p<0,001)$;

- $42,9 \%$ of patients observed for psychomotor agitation $(n=15)$ had a final diagnosis of Delirium or Dementia;

- $53,8 \%$ of patients with a final diagnosis of Psychotic Disorder $(n=7)$ were observed for a history of a previous psychiatric diagnosis.

\section{Conclusions}

- Despite having significant psychopathology requiring intervention, Serious Mental Illness was uncommon among inpatients referred to liaison psychiatry;

- Adjustment Disorders were the most common diagnosis, which is congruent with a population of patients hospitalized with serious health issues;

- Observation of patients with Psychotic Disorders was most commonly requested only because of their psychiatric diagnosis, suggesting lack of confidence of non-psychiatry doctors on dealing with these patients without a previous psychiatric evaluation;

- Almost half of the observed cases of psychomotor agitation did not have a primary psychiatric underlying cause;

- A timely observation of these patients by the Liaison Psychiatry team allows an adequate management and referral to outpatient monitoring. 